\title{
Two-phase flow modelling within expansion and contraction singularities
}

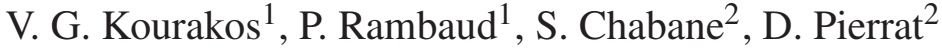 \\ \& J. M. Buchlin ${ }^{1}$ \\ ${ }^{1}$ von Karman Institute for Fluid Dynamics, Belgium \\ ${ }^{2}$ Centre Technique des Industries Mécaniques, France
}

\begin{abstract}
An experimental study is performed in order to describe the single- and twophase (air-water) horizontal flow in the presence of pipe expansion and contraction. Three types of singularities are investigated; smooth convergence and sudden and progressive enlargement. The opening angles for progressive singularities are 5, 8 , 9 and 15 degrees. The surface area ratios tested are $\sigma=0.43,0.64,0.65$ and 1.56. Bubbly flow is the dominant flow regime that is investigated for volumetric quality up to $30 \%$. The pressure distribution for both single and two-phase horizontal flow is examined versus axial position. For expansion geometries, it is found that the smaller the enlargement angle, the larger the recovery pressure for the same flow conditions; the pressure drop caused by the singularity is higher in the case of a sharper expansion. The comparison of the experimental results to published models leads to a proposed corrective coefficient for Jannsen's correlation. Flow visualization is also performed; the flow patterns downstream from the different singularities are identified in each configuration and plotted in Baker's map for horizontal flow.
\end{abstract}

Keywords: two-phase flow, singularity, sudden expansion, contraction, pressure drop, bubbly flow, flow visualization.

\section{Introduction}

Two-phase flow can be frequently met in nuclear, chemical or mechanical engineering where gas-liquid reactors, boilers, condensers, evaporators and combustion systems are often used. The presence of geometrical singularities in pipes may significantly affect the behaviour of two-phase flow and subsequently the 
resulting pressure drop. Therefore, it is an important subject of investigation in particular when the application concerns industrial safety valves. The studies of two-phase flow in straight pipes existing in the literature are numerous. However, investigations of two-phase flow in divergence, convergence, bends and other types of singularities are rather sparse. The aim of studying these geometries is to find how these geometrical accidents influence the two-phase flow pattern and pressure distribution. In particular, the understanding of the flow in such basic geometries can lead to a better design of safety systems.

Some of the authors that have analyzed two-phase flow in expansion geometries are Jannsen and Kervinen [1], McGee [2], Chisholm [3], Chisholm [4] and Lottes [5]. Correlations for estimating the pressure change in two-phase flow in this type of piping geometry are reported by these authors. These correlations can be extracted from the conservation equations applied downstream of the sudden expansion. The equations used take into account different parameters of the geometry and the flow such as surface area ratio $\sigma$, mass quality $x$ and mass velocity $G$. More recently, Aloui and Souhar [6], Aloui et al. [7], Schmidt and Friedel [8], Hwang and Pal [9], et al. Ahmed et al. [10] and Ahmed et al. [11] have evaluated the pressure change in a sudden expansion duct. Moreover, some of them (Aloui and Souhar [6]; Ahmed et al. [10]) have measured the bubble velocities and local void fraction to characterize the flow regime downstream from the singularity. The lack of studies in progressive enlargements in two-phase flow in the literature makes such an investigation more appealing. In this paper, progressive contraction and divergence geometry of different opening angles is considered. The latter is compared to the case of sudden expansion. The two fluids are air and water in isothermal conditions. The volumetric quality of the air varies from $0-30 \%$ and bubbly flow is the dominant regime. Four surface area ratios, $\sigma=0.43,0.64,0.65$ and 1.56 , are tested. The opening angles for the case of progressive singularities are 5, 8, 9 and 15 degrees. The Reynolds number $R e$ of the liquid ranges from $8 \cdot 10^{4}$ to $23 \cdot 10^{4}$. The determination of the recovery pressure for each of the aforementioned geometries is one of the main objectives of this investigation.

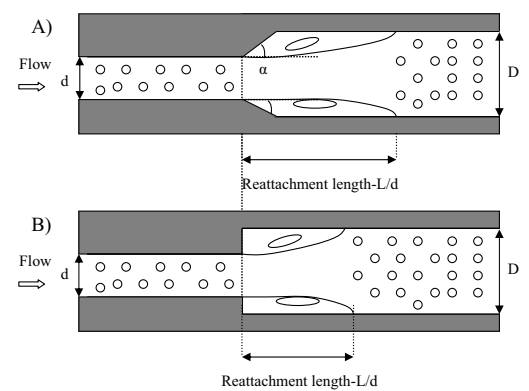

Figure 1: A) Progressive expansion of different opening angles-reattachment length L/d. B) Sudden expansion-reattachment length L/d. 
In Figure 1, the two different types of expansion geometries tested in this paper are presented. Figure 1A shows the divergent pipe with the opening angle $\alpha$ and Figure $1 \mathrm{~B}$ the sudden expansion. The normalized reattachment length L/d, noticed in Figure 1, denotes the eventual recirculation zone. In the case of convergence geometry, a contraction region can be observed; a vena contracta is formed in the pipe downstream from the singularity.

\section{Experimental facility and conditions}

\subsection{Experimental facility}

A schematic of the horizontal air-water flow facility used for the present study is shown in Figure 2. A centrifugal pump (1) with a maximum flow rate of $65 \mathrm{~m}^{3} / \mathrm{h}$ is sucking water from a reservoir and is controlled with a frequency inverter. During the experiments, an air release valve (11) connected to the tank is kept continuously open to the atmosphere to avoid bubbles entering the circuit. A by pass valve (12) is used to prevent facility from the water hammer phenomenon. A temperature sensor is placed in the reservoir to monitor the temperature for each measurement. Two electronic flow meters are used to measure the water flow rate ( 2 and 3 ); their maximum capacity is $12 \mathrm{~m}^{3} / \mathrm{h}(3)$ and $32 \mathrm{~m}^{3} / \mathrm{h}(2)$, respectively. In the case of the desired maximum flow rate, which is $40 \mathrm{~m}^{3} / \mathrm{h}$, the two flow meters are used in series. A bourdon tube pressure gauge (4) is placed upstream in the pipe to obtain the wall static pressure relative to the atmosphere. This indication helped to prevent excessive pressure that could lead to a breaking of the test section (made in Polymethyl Methacrylate, PMMA). Moreover, the pressure has to be high enough to allow the necessary purging of the pressure transducers. Therefore, the pressure is held constant at around $200 \mathrm{kPa}$. The setup has an upstream calming section (5) consisting of a stainless steel pipe length of 50 diameters (50d). This ensures a fully developed flow after the bend. Close to the test section, the injection of the air is performed through a gas injector (6) as indicated in Figure 2. A regulation valve (7)

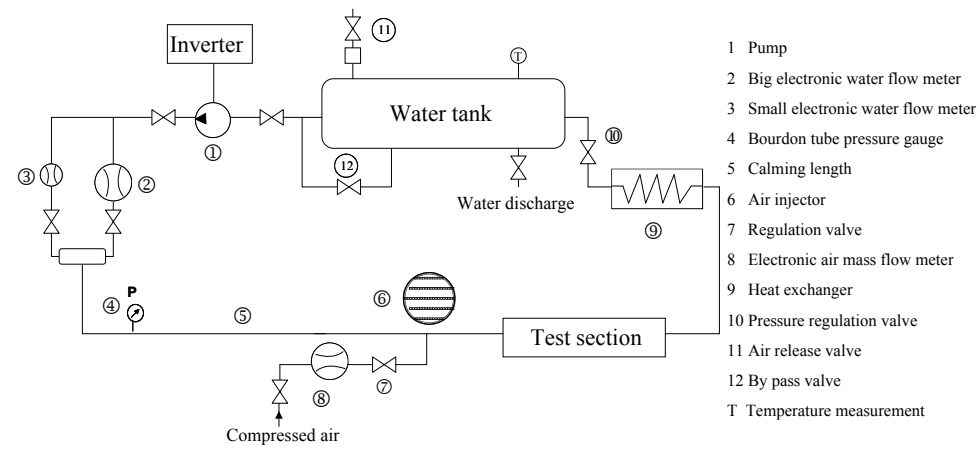

Figure 2: Schematic of the experimental facility. 
controls the air that is supplied from a compressor. The air flow rate is measured by an electronic mass flow meter (8). The design and the positioning of the air injection devices are such that uniform bubbly flow is produced at the inlet of the test section. It is found that the most suitable distance for the air injection is 20 pipe diameters upstream from the singularity. After the test section, a heat exchanger (9) is placed for maintaining the temperature constant at around $21^{\circ} \mathrm{C}$ during the experiments. A draining valve is also located at the bottom of the reservoir. Finally, a pressure regulation valve (10) controls the pressure of the system.

A detailed view of the test section is presented in Figures 3, 4 and 5. The case of a DN 40/65 ( $\sigma=0.43)$ divergent section with an opening angle of $8^{\circ}$ is exemplified. At each section of measurement, four pressure taps are placed with an angle of $45^{\circ}$ between them as shown in Figure 3. Thus, any three dimensionality of the flow could be identified from pressure measurement. The four taps are named as A, B, C and $\mathrm{D}$ according to Figure 3. Figure 4 depicts an overview of the test section. The setup is built in PMMA to allow optical access. Pressure taps are placed along the tube in several points as is shown in Figure 5. The distance between pressure holes is normally equal to one tube diameter but becomes smaller when approaching the singularity. The pressure taps are also more dense inside and downstream from the singularity. This allows better tracking of the flow behaviour in the singularity.

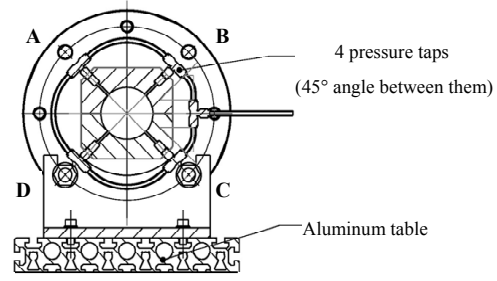

Figure 3: Pressure taps placed in four different points of the tube with $45^{\circ}$ between them.

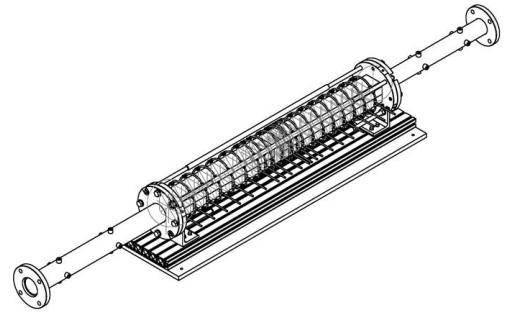

Figure 4: Overview of the test section.

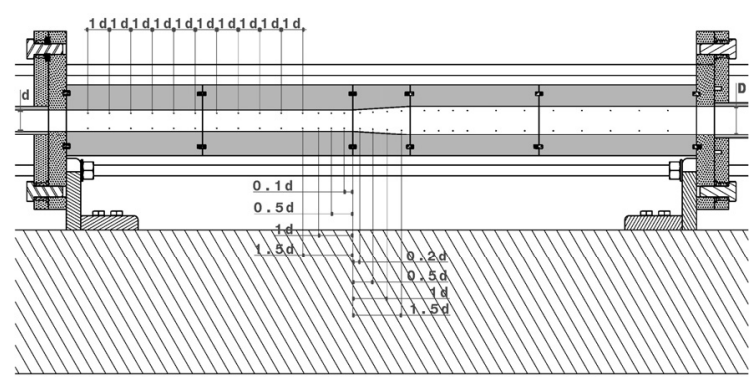

Figure 5: Detailed view of the test section with the pressure taps and their position. 
Table 1: Different test cases studied.

\begin{tabular}{|c|c|c|c|c|c|c|}
\hline Singularity & $d_{1}[\mathrm{~m}]$ & $D_{2}[\mathrm{~m}]$ & $\sigma[-]$ & $L_{s}[\mathrm{~m}]$ & $L_{S} / d_{1}$ & Angle $\alpha\left[^{\circ}\right]$ \\
\hline Smooth contraction & 0.04 & 0.032 & 1.56 & 0.025 & 0.63 & 9 \\
\hline Divergence & 0.032 & 0.04 & 0.64 & 0.025 & 0.78 & 9 \\
\hline Divergence & 0.041 & 0.0627 & 0.43 & 0.041 & 1 & 15 \\
\hline Divergence & 0.041 & 0.0627 & 0.43 & 0.07503 & 1.83 & 8 \\
\hline Divergence & 0.041 & 0.0627 & 0.43 & 0.1238 & 3 & 5 \\
\hline Divergence & 0.0627 & 0.078 & 0.65 & 0.0529 & 0.8 & 8 \\
\hline Sudden expansion & 0.041 & 0.0627 & 0.43 & - & - & 90 \\
\hline Sudden expansion & 0.0627 & 0.078 & 0.65 & - & - & 90 \\
\hline
\end{tabular}

Table 2: Upstream conditions for pressure measurements and flow visualization.

\begin{tabular}{|c|c|c|c|c|c|c|c|c|}
\hline$d_{1}[\mathrm{~m}]$ & Fluid & $\bar{Q}[1 / \mathrm{s}]$ & $J[\mathrm{~m} / \mathrm{s}]$ & $\beta[\%]$ & $\bar{G}\left[\mathrm{~kg} / \mathrm{m}^{2} \mathrm{~s}\right]$ & $\mathrm{Re}_{L 1} \cdot 10^{4}$ & Flow regime & \\
\hline \multirow{5}{*}{0.032} & Water & 2 & 2.5 & & 2500 & 9 & Laminar & Min \\
& Water & 4.7 & 5.8 & $1-40$ & 5850 & 20 & Turbulent & Max \\
& Air & 0.017 & 0.02 & & 0.03 & 0.005 & Laminar & Min \\
& Air & 1.8 & 2.2 & & 2.61 & 0.46 & Turbulent & Max \\
\hline \multirow{5}{*}{0.041} & Water & 2.3 & 1.8 & & 1750 & 8 & Turbulent & Min \\
& Water & 7 & 5.4 & $5-30$ & 5300 & 23 & Turbulent & Max \\
& Air & 0.4 & 0.3 & & 0.38 & 0.09 & Laminar & Min \\
& Air & 2.8 & 2.2 & & 2.73 & 0.58 & Turbulent & Max \\
\hline \multirow{5}{*}{0.0627} & Water & 6 & 1.9 & & 1950 & 13 & Turbulent & Min \\
& Water & 10.5 & 3.4 & $5-25$ & 3400 & 23.5 & Turbulent & Max \\
& Air & 0.4 & 0.1 & & 0.15 & 0.05 & Laminar & Min \\
& Air & 3.4 & 1.1 & & 1.29 & 0.45 & Turbulent & Max \\
\hline
\end{tabular}

Pressure distribution is measured upstream and downstream from the divergence. The test matrix is summarized in Table 1.

\subsection{Flow conditions and measurement devices}

The flow conditions of the experimental campaigns are listed in Table 2. Table 2 presents the test conditions for the pressure measurements and for flow visualization. It should be pointed out that the $\operatorname{Re}_{L 1}$ number of the liquid is based on the upstream pipe diameter d. For the comparison between single and two-phase flow, $\operatorname{Re}_{L 1}$ is kept constant. This is obtained by adjusting the water flow rate when increasing the air to reach a higher volumetric quality $\beta$. Consequently, we can assume that the total mass flux is constant, since the mass of the air compared to that of water is negligible. Differential pressure transducers of the Rosemount type are used. The uncertainty associated with the pressure transducers varies from a minimum of $0.35 \%$ to a maximum of $0.75 \%$, depending on the range of the 
measurement (100-20\% of the scale of the range respectively). To obtain the best accuracy possible, four different pressure transducers are selected:

1. Calibrated at $0-1.6 \mathrm{kPa}$

2. Calibrated at $0-4 \mathrm{kPa}$

3. Calibrated at $0-8 \mathrm{kPa}$

4. Calibrated at $0-16 \mathrm{kPa}$

Every transducer is used in a range that gives the best accuracy in all the conditions covered. Prior to the measurements, predictions of regular pressure drop are performed by means of Blasius and Colebrook-White formulas for single-phase and Lockhart and Martinelli [12] for two-phase flow. Thus, this $\Delta \mathrm{P}$ estimation allows the selection of the appropriate pressure transducers for each test. Additionally, for the prediction of the singular pressure change in singlephase, the coefficients given by Idel'cik [13] are used. The uncertainty related to the flow rate measurements varies from a minimum of $0.5 \%$ to a maximum of $1.10 \%$. The temperature variation during the experiments is of the order of $\pm 4^{\circ} \mathrm{C}$ with an average value of $21^{\circ} \mathrm{C}$. Although a heat exchanger is used for reducing this variation, a small fluctuation of the temperature could not be avoided. A variation of $\pm 5^{\circ} \mathrm{C}$ will change $\rho$ and $v$ by $0.1 \%$ and $11 \%$ respectively. Therefore, a correction of the liquid density and viscosity is performed. The sampling frequency of the measurements is $f_{\text {sampling }}=2 \mathrm{~Hz}$ and the acquisition time for each measurement point is $t_{a c q}=1$ minute with the aim of assuring a more accurate average. In some cases (for sudden and progressive enlargement of $\sigma=0.65$ ), a higher fluctuation of the signal is observed; in this occurrence an acquisition time of 2 minutes is chosen.

\section{Results and discussion}

\subsection{Pressure measurements}

One of the main objectives of the study is the determination of the pressure distribution through the different singularities. Figure 6 indicates how the measurements are performed and how the singular single and two-phase pressure change is determined (the case of divergence is chosen). As the graph of Figure 6 shows, following a normal decrease upstream from the geometrical accident, the pressure will increase to a maximum value inside the divergent section and will start decreasing after a certain length in a regular way. We can split the whole phenomenon into three regions; the upstream fully developed flow, the transitional region with a recirculation zone and the downstream fully developed flow. The length of the transitional region varies with $\operatorname{Re}_{L 1}, \sigma$, and the type of the singularity. In all the tests, the measurement of the regular and singular static pressure changes refers to the pressure measured at $\approx 10 \mathrm{~d}$ upstream from the singularity (Figure 6). The singular pressure change $\Delta \mathrm{P}$ can be finally determined by extrapolating the regular static pressure drop from the start of the singularity to the reattachment point. Since the points downstream from the singularity are not enough to obtain fully established flow, the regular pressure drop is 


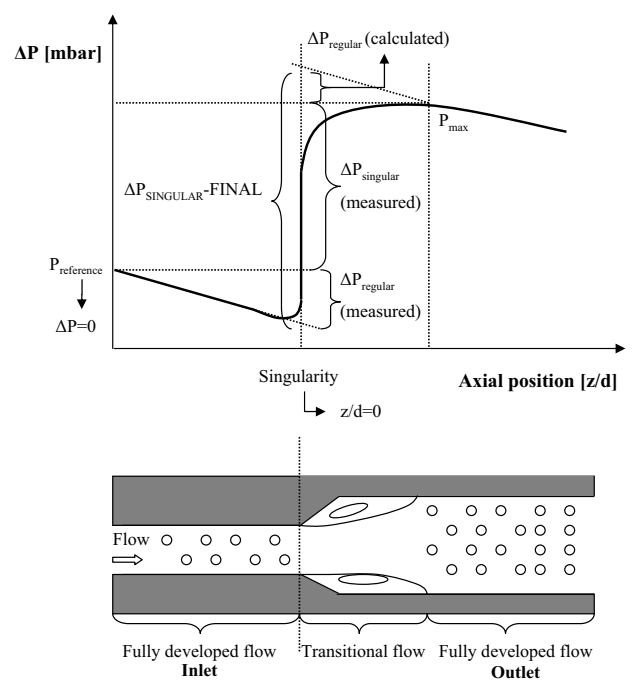

Figure 6: Explanation of the way to determine the singular pressure change in expansion geometry.

calculated by means of the Blasius and Colebrook-White formulas for singlephase and the model of Lockhart and Martinelli [12] for two-phase. The final singular pressure change is calculated by a simple summation of these three terms $\left(\left|\Delta P_{\text {regular-measured }}\right|+\left|\Delta P_{\text {singular-measured }}\right|+\left|\Delta P_{\text {regular-calculated }}\right|\right)$. The reattachment length is determined as the location of the maximum recovery pressure.

\subsubsection{Expansion singularities}

3.1.1.1 Sudden expansion In Figure 7, the two-phase pressure change along the pipe and the singularity is plotted for sudden expansion of $\sigma=0.43$ and at $\operatorname{Re}_{L 1}=1.82 \cdot 10^{5}$. The single-phase result is also drawn on the same graph. The pressure is measured at the four peripheral taps on the tube sections close to the singularity (points $\mathrm{A}, \mathrm{B}, \mathrm{C}$ and D) as well as their average (point $\mathrm{M}$ ). The two-phase experimental data are compared with prediction of the singular pressure change for axisymmetric sudden expansion geometry obtained from the two following models:

Jannsen and Kervinen [1]:

$$
\Delta P_{t o t}=-\frac{G_{1}^{2}}{2 \rho_{L}}(1-\sigma)^{2}\left[1+x\left(\frac{\rho_{L}}{\rho_{G}}-1\right)\right]
$$

where $G_{1}$ is the mass flux upstream from the singularity, $\rho_{L}$ is the density of water, $\sigma$ is the area ratio, $x$ is the mass quality of air and $\rho_{G}$ is the density of air. 


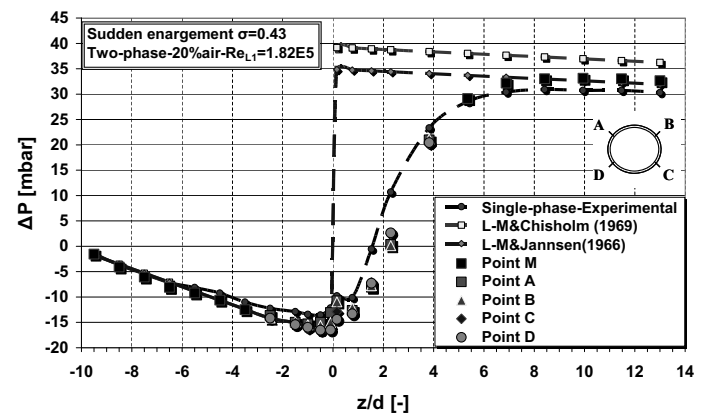

Figure 7: Two-phase static pressure change versus axial position for sudden enlargement of $\sigma=0.43$ and for $\operatorname{Re}_{L 1}=1.82 \cdot 10^{5}$-comparison with experimental single-phase and with the models of Jannsen and Kervinen [1] and Chisholm [4].

Chisholm [4]:

$$
\Delta P_{s t}=-\frac{G_{1}^{2}}{2 \rho_{L}} \sigma(1-\sigma)(1-x)^{2}\left(1+\frac{C}{X}+\frac{1}{X^{2}}\right),
$$

where

$$
\begin{gathered}
X^{2} \triangleq\left(\frac{1-x^{2}}{x}\right) \frac{\rho_{G}}{\rho_{L}} \\
C=\left[1+0.5\left(\frac{\rho_{L}-\rho_{G}}{\rho_{L}}\right)^{0.5}\right]\left[\left(\frac{\rho_{L}}{\rho_{G}}\right)^{0.5}+\left(\frac{\rho_{G}}{\rho_{L}}\right)^{0.5}\right] .
\end{gathered}
$$

Both models rely on the assumption of a homogeneous flow. Figure 7 shows that Jannsen's model [1] fits satisfactorily with the experimental results while Chisholm's [4] model overestimates the pressure change. This was also reported by Velasco [14].

To better emphasize the effect of two-phase flow we define the dimensionless pressure change $\Phi_{L}$ as follows:

$$
\Phi_{L}=\frac{\Delta P_{\text {Singular }}^{T P}}{\Delta P_{\text {Singular }}^{S P}}
$$

where $\triangle P_{\text {Singular }}^{T P}$ is the singular two-phase pressure change as explained in Figure 6 and $\Delta P_{\text {Singular }}^{S P}$ the single-phase one. Figure 8 displays the evolution of the experimental $\Phi_{L}$ versus volumetric quality at $\operatorname{Re}_{L 1}=2.0 \cdot 10^{5}$. The data are compared to the model of Jannsen and Kervinen [1] and Chisholm [4], respectively. As it was previously mentioned, Jannsen's [1] correlation agrees better than Chisholm's [4] correlation with the experimental results. The comparative graphs given in Figures 9 and 10 indicate that the maximum deviation from the 


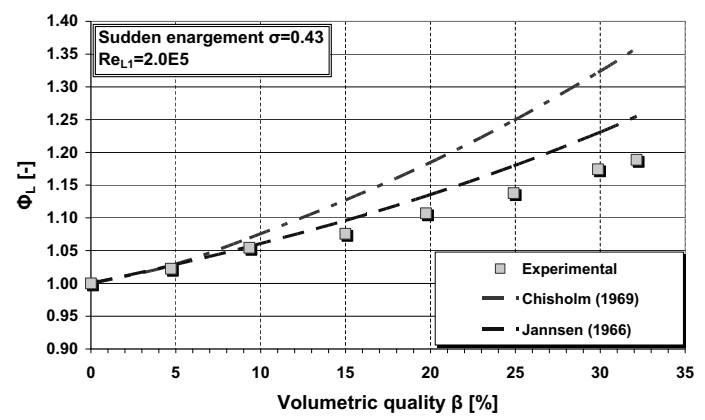

Figure 8: Dimensionless singular pressure change L versus volumetric quality. Comparison with the models of Jannsen and Kervinen [1] and Chisholm [4].

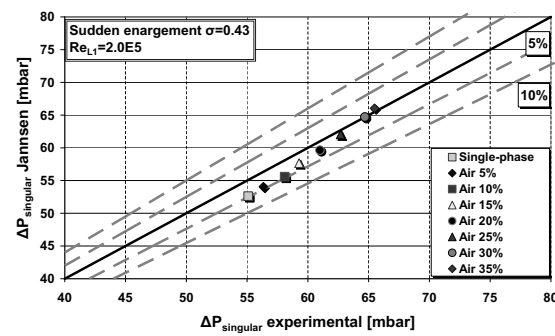

Figure 9: Deviation of Jannsen and Kervinen [1] model from experimental results.

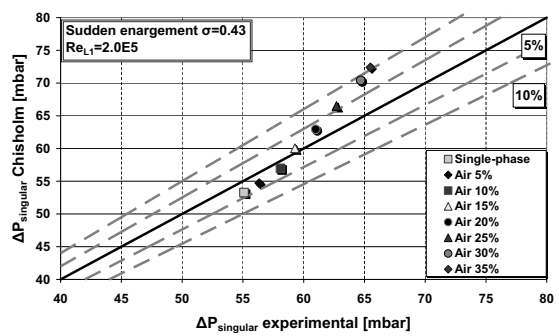

Figure 10: Deviation of Chisholm [4] model from experimental results.

experimental data for the model of Jannsen and Kervinen [1] is limited to 5\% while it reaches $10 \%$ for Chisholm [4] model.

Measurements with the same flow conditions are repeated for a sudden enlargement of surface area ratio $\sigma=0.65$. A summarizing graph of the static pressure recovery measured in both geometries of $\sigma=0.43$ and 0.65 for different $\operatorname{Re}_{L 1}$ and for volumetric quality, $\beta$, varying from 0 to $35 \%$ is presented in Figure 11 . The singular pressure change is increasing for higher $\beta$ and $\operatorname{Re}_{L 1}$. Furthermore, for the same $\operatorname{Re}_{L 1}$ lower $\sigma$ results in a lower $\Delta P$ (up to three times smaller).

3.1.1.2 Progressive and sudden enlargement: comparison Compared to sudden expansion, a progressive enlargement will create for the same flow conditions, less pressure loss and accordingly will exhibit a higher pressure recovery as depicted in Figures 12 and 13. Figure 12 shows a single-phase $\Delta \mathrm{P}$ diagram along sudden expansion and divergent of angles 5, 8 and $15^{\circ}$, of surface area ratio $\sigma=0.43$ and at $\operatorname{Re}_{L 1}=1.8 \cdot 10^{5}$. In Figure 13, the same type of plot is built for $\beta=20 \%$ of air. It can be seen that, for single-phase, the pressure drops $17 \%$ passing from divergent section of $5^{\circ}$ to $15^{\circ}$ and $29 \%$ from $5^{\circ}$ to sudden 


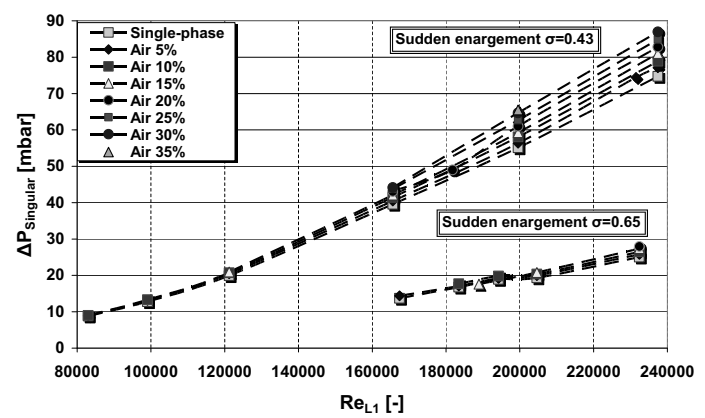

Figure 11: $\Delta P_{\text {singular }}$ for several $\operatorname{Re}_{L 1}$ from $0-35 \%$ of air for sudden enlargement of surface areas $\sigma=0.43$ and $\sigma=0.65$.

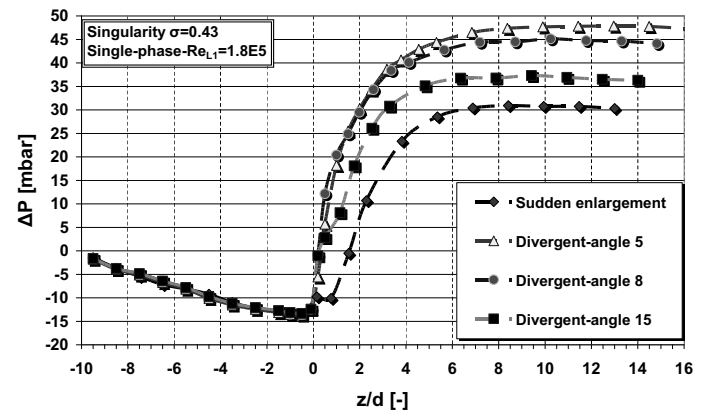

Figure 12: Pressure recovery diagram for single-phase flow and the same $\operatorname{Re}_{L 1}$, a singularity of $\sigma=0.43$ and for sudden enlargement and divergence of angles 5,8 and $15^{\circ}$.

expansion. For two-phase flow, the pressure drop is $11 \%$ and $21 \%$ respectively. Additionally, we can notice that all the curves in Figure 13 are shifted to the right, meaning that the flow becomes fully developed further downstream from the singularity and thus the recirculation zone is longer in two-phase flow. In the case of sudden enlargement, contrary to smooth divergence, the pressure before starting to increase slightly decreases at $1 \mathrm{~d}$ and starts increasing again at $2 \mathrm{~d}$ upstream of the singularity. This is due to the presence of a secondary recirculation zone.

3.1.1.3 Proposed correlation for expansion singularities The proposed correlation relies upon Jannsen [1] formulation. By fitting this model to the experimental values, a corrective coefficient is defined. It turns out that this parameter $C$ is a function of the opening angle $\alpha$ and $\operatorname{Re}_{L 1}$ as shown by the $3 D$ representation proposed in Figure 14. Although Jannsen's [1] model is chosen as the most accurate, attempts are made with Chisholm [4] model as well. Hence, the corrective coefficient $C$ for Chisholm's [4] correlation is represented in a $3 D$ plot 


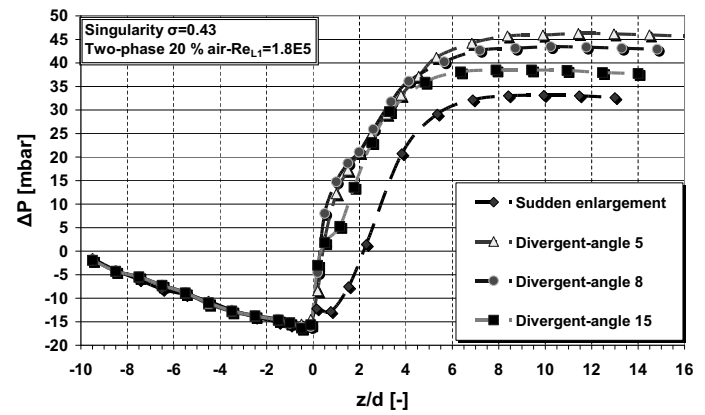

Figure 13: Pressure recovery diagram for two-phase flow (20\% of air) and the same $\operatorname{Re}_{L 1}$, a singularity of $\sigma=0.43$ and for sudden enlargement and divergence of angles 5,8 and $15^{\circ}$.

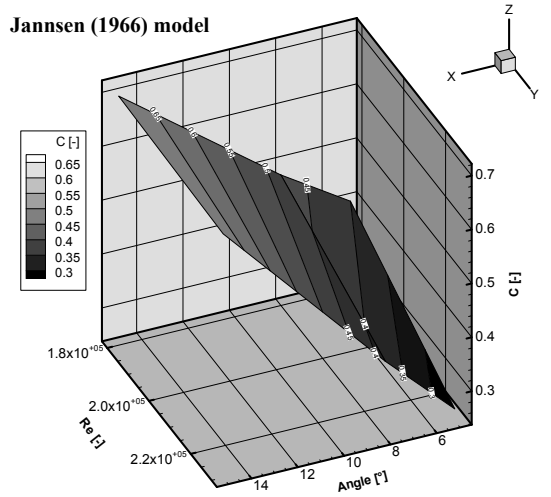

Figure 14: Coefficient $C$ in function of $\alpha$ and $\operatorname{Re}_{L 1}$ for Jannsen and Kervinen [1] model.

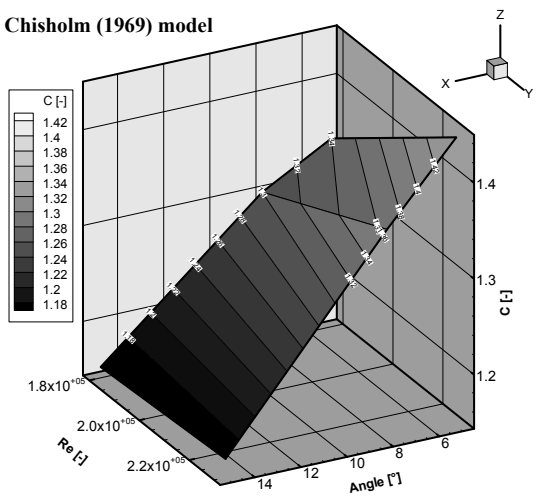

Figure 15: Coefficient $C$ in function of $\alpha$ and $\operatorname{Re}_{L 1}$ for the Chisholm [4] model.

in Figure 15. In Table 3, the coefficients that are calculated for both models and for the different parameters tested in progressive expansion are given.

The corrective coefficient $C$ for Jannsen and Kervinen [1] formulation can be modelled as follows:

$$
C=0.061 \cdot \alpha^{0.8917}-10717 \cdot \operatorname{Re}_{L 1}^{-0.8283}+0.378
$$

This coefficient when applied to Jannsen's [1] model gives a maximum deviation from the model fit of $58 \%$ for the case of $\sigma=0.43, \operatorname{Re}_{L 1}=1.84 \cdot 10^{5}$ and $5^{\circ}$ and minimum of $1.4 \%$ for $\operatorname{Re}_{L 1}=2.36 \cdot 10^{5}$ and $15^{\circ}$. It should be stressed that further experimental data are needed to refine the $C$ modelling and improve the validation. 
Table 3: Coefficient for adaption of the Jannsen and Kervinen [1] and Chisholm [4] models to fit to the experimental results for divergence geometry and for several $\alpha, \sigma$ and $\operatorname{Re}_{L 1}$.

\begin{tabular}{|c|c|c|c|c|c|c|c|}
\hline \begin{tabular}{|l|} 
Jannsen [1] \\
\end{tabular} & $\operatorname{Re}_{L 1} \cdot 10^{5}$ & $\alpha\left[^{\circ}\right]$ & $C[-]$ & Chisholm [4] & $\operatorname{Re}_{L 1} \cdot 10^{5}$ & $\alpha\left[^{\circ}\right]$ & $C[-]$ \\
\hline \multirow{6}{*}{$\sigma=0.43$} & 1.84 & 5 & 0.4 & \multirow{6}{*}{$\sigma=0.43$} & 1.84 & 5 & 1.34 \\
\hline & 2.3 & 5 & 0.26 & & 2.3 & 5 & 1.445 \\
\hline & 1.78 & 8 & 0.48 & & 1.78 & 8 & 1.3 \\
\hline & 2.36 & 8 & 0.38 & & 2.36 & 8 & 1.365 \\
\hline & 1.76 & 15 & 0.7 & & 1.76 & 15 & 1.155 \\
\hline & 2.36 & 15 & 0.69 & & 2.36 & 15 & 1.163 \\
\hline \multirow{2}{*}{$\sigma=0.65$} & 1.79 & 8 & 0.3 & \multirow{2}{*}{$\sigma=0.65$} & 1.79 & 8 & 1.187 \\
\hline & 2.26 & 8 & 0.24 & & 2.26 & 8 & 1.365 \\
\hline
\end{tabular}

Finally, the modified Jannsen's [1] correlation can be written as:

$$
\begin{aligned}
\Delta P_{\text {tot }} & =-\left[0.061 \cdot \alpha^{0.8917}-10717 \cdot R e_{L 1}^{-0.8283}+0.378\right] \\
& \cdot \frac{G_{1}^{2}}{2 \rho_{L}}(1-\sigma)^{2}\left[1+x\left(\frac{\rho_{L}}{\rho_{G}}-1\right)\right] .
\end{aligned}
$$

\subsubsection{Contraction singularity}

3.1.2.1 Measurements in progressive contraction Convergence geometry of $\sigma=1.56$ and angle $9^{\circ}$ is studied. The geometry is identical to the test section shown in Figure 5 with a scaling factor of $1 / 2$ (DN40/32). The experimental facility and flow conditions are described in section 2.2. Pressure transducers of type Validyne are used for this experimental campaign with the same acquisition time $\left(\mathrm{t}_{\text {acq }} .=1 \mathrm{~min}\right)$ and sampling frequency $\left(\mathrm{f}_{\text {sampling }}=2 \mathrm{~Hz}\right)$. The different membranes that cover all the range of the pressure measurements are:

1. Calibrated at $0-2.2 \mathrm{kPa}$

2. Calibrated at $0-8.6 \mathrm{kPa}$

3. Calibrated at $0-35 \mathrm{kPa}$

Additionally, numerical simulations are carried out with the commercial CFD code Fluent. The test parameters and conditions are: $2 D$ axisymmetric computation, realizable $k-\epsilon$ turbulence model with enhanced wall treatment and second order discretization scheme. Convergence criterion is set at $10^{-7}$. In Figure 16, the experimental and numerical static pressure drop is plotted against axial position for several $\operatorname{Re}_{L 1}$ in single and two-phase flow. The pressure is decreasing in a regular way before the singularity; the contraction creates a high pressure drop step and then starts decreasing regularly downstream.

The flow is observed fully developed close to the singularity (at $\approx 2 \mathrm{~d}$ upstream and downstream) contrary to the case of divergence for which the reattachment length is detected at $\approx 10 \mathrm{~d}$. Therefore, the singular pressure change $\Delta \mathrm{P}_{\text {singular }}$ for convergence geometry is determined by measuring the static pressure at equal 
distance upstream and downstream from the singularity (2d). A summarizing graph of all experimental and numerical results obtained for single and two-phase flow is shown in Figure 17. The results concerning the case of sudden contraction for several $\sigma$ and $G$ (Guglielmini et al. [15]) are compared to the experimental data. The experimental results for smooth contraction are plotted in terms of the dimensionless pressure change $\Phi_{L}$, defined by eqn. (3). In Figure 17 Jannsen's [1] correlation for sudden contraction is adapted with a correction coefficient of $C=0.81$ to fit with the results $\left(G=1990 \mathrm{~kg} / \mathrm{m}^{2} \mathrm{~s}\right)$.

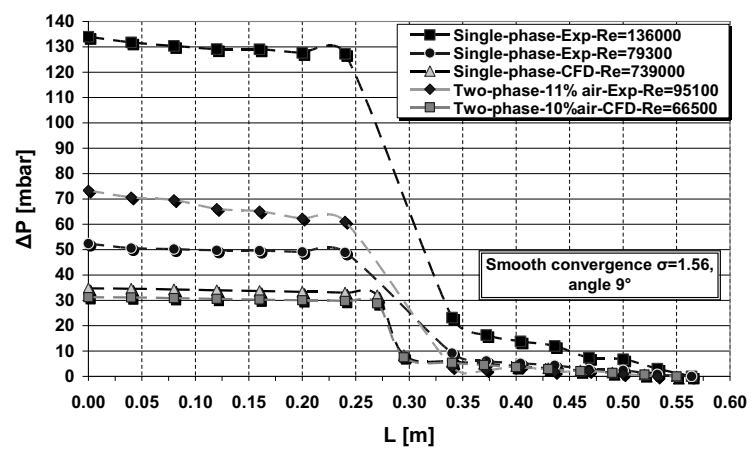

Figure 16: Experimental and numerical single and two-phase static pressure change versus axial position for convergence of $\sigma=1.56$ and angle $9^{\circ}$ for several $\operatorname{Re}_{L 1}$.

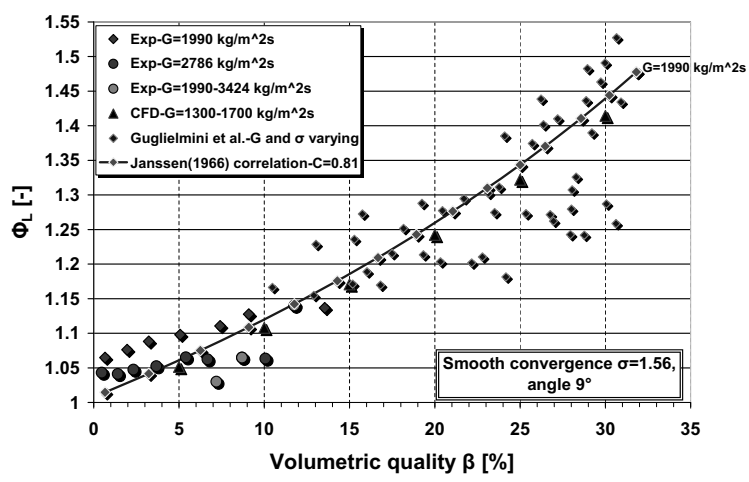

Figure 17: Experimental and numerical dimensionless singular pressure change $\Phi_{L}$ versus volumetric quality. Comparison to literature (Guglielmini et al. [15]) and to the adapted $(C=0.81)$ Jannsen and Kervinen [1] model. 
Table 4: Coefficient for adaption of Jannsen's [1] formulation to fit to the experimental and numerical results for progressive contraction for several $G$.

\begin{tabular}{|c|c|c|}
\hline Jannsen [1] correlation & $\mathbf{G}_{L 1}\left[\mathrm{~kg} / \mathbf{m}^{2} \mathbf{s}\right]$ & Correction coefficient $C[-]$ \\
\hline & 1592 & 0.835 \\
& 1990 & 0.800 \\
Convergence-angle $9^{\circ}, \sigma=1.56$ & 2786 & 0.770 \\
& 4378 & 0.754 \\
\hline
\end{tabular}

3.1.2.2 Proposed correlation for progressive contraction The correlation for sudden convergence, as described from Jannsen [1], is recalled:

$$
\Delta P_{T P}=\frac{G_{2}^{2}}{2 \rho_{L}}\left(\left(\frac{1}{C_{C}}-1\right)^{2}+1-\frac{1}{\sigma^{2}}\right)\left[1+x\left(\frac{\rho_{L}}{\rho G}\right)\right] .
$$

where $C_{c}$ is the contraction coefficient defined as $C_{c}=A_{c} / A_{1}$ where $A_{c}$ the flow area in the vena contracta. A typical value of this parameter equal to 0.64 is considered for this investigation. This correlation can be modified and then applied for the case of smooth contraction. The parameter varying is the mass flux of water upstream of the singularity $G_{L 1}$. A fit to the present results is made and the resulting corrective coefficients are listed in Table 4.

A correlation to calculate the correction coefficient $C$ is obtained as a function of $G_{L 1}$.

$$
C=2 \cdot 10^{-8} G_{L 1}^{2}-0.0001 \cdot G_{L 1}+0.9913 .
$$

The relative discrepancy between experimental-numerical data and model fit, when eqn. (7) is applied, varies from $5.72 \%$ to a maximum of $24.25 \%$. The final corrected correlation for the case of smooth convergence of angle $9^{\circ}$ is:

$$
\begin{aligned}
\Delta P_{T P} & =\left[2 \cdot 10^{-8} G_{L 1}^{2}-0.0001 \cdot G_{L 1}+0.9913\right] \\
& \cdot \frac{G_{2}^{2}}{2 \rho_{L}}\left(\left(\frac{1}{C_{C}}-1\right)^{2}+1-\frac{1}{\sigma^{2}}\right)\left[1+x\left(\frac{\rho_{L}}{\rho G}\right)\right] .
\end{aligned}
$$

\subsection{Flow pattern maps and visualization}

Flow regime maps are often considered in two-phase flow. A common chart is the one proposed by Baker [16]. It has been established for horizontal flows in pipes of constant cross section. In the present study, the flow is visualized both upstream and downstream from the singularity. As it is illustrated in Figure 18, four different flow patterns are identified downstream of the divergence; Bubbly, Plug, Disperse and Annular flow.

For sudden and progressive enlargement (angles $5^{\circ}$ and $8^{\circ}$ ) with $\sigma=0.43$ and $\sigma=0.65$, a normal video camera is used to determine the condition for transition 
from bubbly flow to other types of flow just after the singularity. The results are plotted on Baker's map and are reported in Figure 19. However, since the departure from bubbly flow is decided on visual information, the transition criterion remains rather subjective and the results given in Figure 19 are only indicative.

The second campaign of visualization is performed, using a high-speed camera, in a fully transparent setup that allows better optical access (without pressure taps). Consequently, distinction between flow regimes is more straightforward. In this facility, a progressive enlargement of $\sigma=0.64$ for an opening angle of $\alpha=9^{\circ}$ is tested. The flow conditions for which these regimes are visualized are reported in Figure 20. Finally, we should draw attention to the fact that all flow conditions calculated refer to the upstream position. Indeed, for these test cases, the flow regime upstream from the singularity corresponds to bubbly flow (Baker map) while downstream three additional flow patterns occur (plug, disperse and annular).

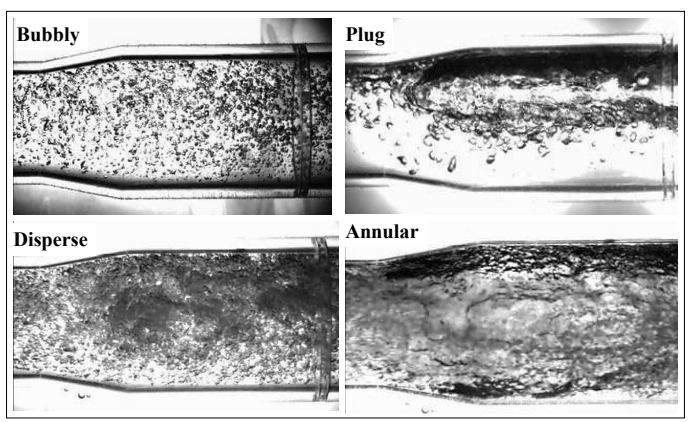

Figure 18: Flow patterns identified downstream of the divergence geometry of $\alpha=$ $9^{\circ}$ and $\sigma=0.64$.

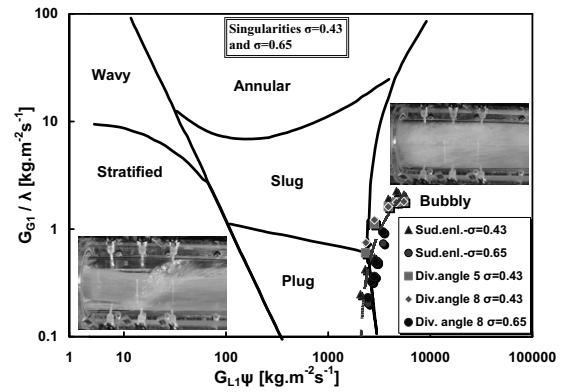

Figure 19: Modified Baker [16] map for progressive and sudden expansion of $\sigma=0.43$ and 0.65 .

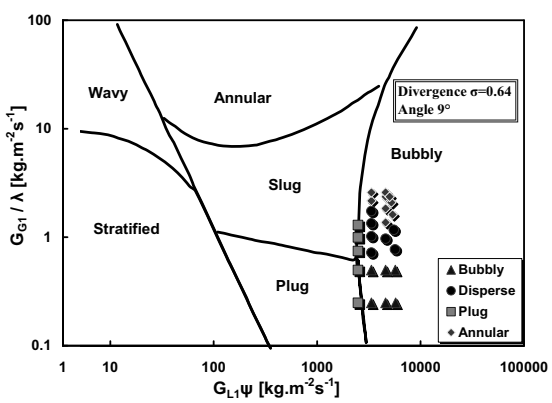

Figure 20: Modified Baker [16] map for progressive expansion of $\sigma=0.64$ and $\alpha=9^{\circ}$. 


\section{Conclusions}

An investigation of horizontal air-water flow in sudden and progressive enlargements and smooth contraction is performed. The static pressure evolution along these geometrical accidents is measured and flow visualization is performed. The results are expressed in terms of the dimensionless singular pressure change $\Phi_{L}$. Compared to literature, a deviation of $5 \%$ is found with Jannsen's [1] model and 10\% with Chisholm's [4] model for axisymmetric sudden expansion. For progressive enlargement of the same surface area ratio $\sigma$, the smallest the opening angle, the highest the pressure recovery. For the same flow conditions, the minimum pressure recovery occurs for sudden enlargement geometry. A modified version of Jannsen's [1] correlation is suggested for both progressive expansion and contraction. A corrective parameter taking into account the different effects of the divergent angle and the liquid Reynolds number of the divergent section and the upstream mass flux for convergence, is introduced. The proposed correlation gives satisfactory results but needs further validation. In the convergence configuration, the single and two-phase static pressure drop along the pipe is compared with literature and CFD simulations; a satisfactory agreement is found. Finally, flow visualization shows that departure from bubbly flow to plug, disperse or annular flow may occur in the downstream section of a divergent singularity.

\section{Acknowledgements}

The support of the French company CETIM (Centre Technique des Industries Mécaniques) is gratefully acknowledged. Mr. E.C. Bacharoudis and Miss R. Delgado-Tardáguila are thanked for their contribution to the numerical and experimental study in this paper.

\section{Nomenclature}

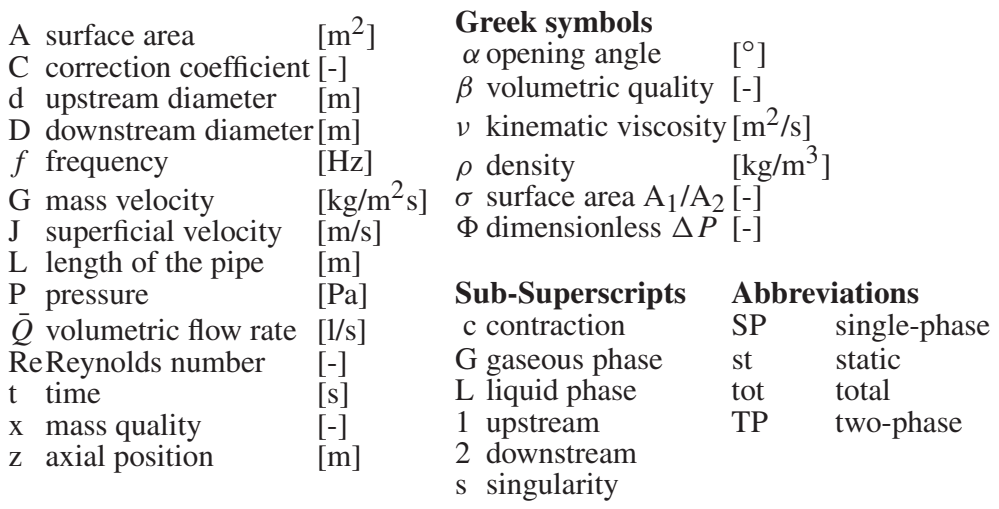




\section{References}

[1] Jannsen, E. \& Kervinen, J.A., Two-phase pressure drop across contractions and expansions of water-steam mixture at 600 to 1400 psia. Technical Report Geap 4622-US, 1966.

[2] McGee, J., Two-phase flow through abrupt expansion and contraction. Ph.D. thesis, North Carolina State University, Raleigh, 1966.

[3] Chisholm, D., Prediction of pressure losses at changes of sections, bends and throttling devices. Technical Report NEL rept. 388, 1968.

[4] Chisholm, D., Theoretical aspects of pressure changes at changes of section during steam-water flow. Technical Report NEL rept. 418, 1969.

[5] Lottes, P., Expansion losses in two-phase flow. Nucl Sci Eng, 9, pp. 26-31, 1960.

[6] Aloui, F. \& Souhar, M., Experimental study of a two-phase bubbly flow in a flat duct symmetric sudden expansion. Part I: Visualization, pressure and void fraction. Int J Multiphase Flow, 4, pp. 651-665, 1996.

[7] Aloui, F., Doubliez, L., Legrand, J. \& Souhar, M., Bubbly flow in an axisymmetric sudden expansion: pressure drop, void fraction, wall shear stress, bubble velocities and sizes. Exp Therm Fluid Sci, 18, pp. 118-130, 1999.

[8] Schmidt, J. \& Friedel, L., Two-phase pressure change across sudden expansions in duct areas. Chem Eng Commun, 141, pp. 175-190, 1996.

[9] Hwang, C.Y. \& Pal, R., Flow of two-phase oil/water mixtures through sudden expansions and contractions. Chem Eng J, 68, pp. 157-163, 1997.

[10] Ahmed, W., Ching, C. \& Shoukri, M., Pressure recovery of two-phase flow across sudden expansions. Int J Multiphase Flow, 33, pp. 579-594, 2008.

[11] Ahmed, W., Ching, C. \& Shoukri, M., Development of two-phase flow downstream of a horizontal sudden expansion. Int $J$ Heat and Fluid Flow, 29, pp. 194-206, 2008.

[12] Lockhart, R.W. \& Martinelli, R.C., Proposed correlation of data for isothermal two-phase two-component flow in pipes. Chem Eng Prog, 45, pp. 39-48, 1949.

[13] Idel'cik, I.E., Memento des pertes de charge. Editions Eyrolles: 61 Bd SaintGermain Paris, 5th edition, 1986.

[14] Velasco, I., L' écoulement diphasique à travers un élargissement brusque, 1975.

[15] Guglielmini, G., Muzzio, A. \& Sotgia, G., The structure of two-phase flow in ducts with sudden contractions and its effects on the pressure drop. Experimental Heat Transfer, Fluid Mechanics and Thermodynamics, 1997.

[16] Baker, O. Oil Gas J, 53, p. 185, 1954. 